\title{
A Micro Device for Separation of Erythrocytes and Leukocytes in Human Blood
}

\author{
Siyang Zheng ${ }^{1}$, Yu-Chong Tai ${ }^{1}$, Harvey Kasdan ${ }^{2}$ \\ ${ }^{I}$ Caltech Micromachining Lab, California Institute of Technology, USA \\ ${ }^{2}$ Iris Diagnostics, International Remote Imaging Systems, Inc., USA
}

\begin{abstract}
We report a MEMS device for continuous and binary separation of leukocytes (white blood cells, WBCs) and erythrocytes (red blood cells, RBCs) by their sizes. The system consists of PDMS cylindrical pillars inside fluidic chamber on glass slides. A $\mathbf{4 2 0} \boldsymbol{\mu m}$ lateral separation was achieved for $5 \mu \mathrm{m}$ and $10 \mu \mathrm{m}$ beads. The critical particle size for separation was successful predicted by simulation and experimentally determined by calibration with polystyrene beads. The separation function (lateral displacement versus particle size) was also measured experimentally. Diluted whole blood and blood fraction of concentrated leukocytes were tested with the devices with erythrocyte to leukocyte ratio in agreement with blood count results. Experiments with fluorescent labeled leukocytes confirm the separation of leukocytes from erythrocytes and measured the separation efficiency.
\end{abstract}

\section{INTRODUCTION}

Recently, there are efforts on cell separation in micro scale using, for examples, field-flow fractionations [1, 2], dielectrophoretic force separation [3], magnetic separator [4]. More related to this work are examples to separate cells based on size, using sedimentation field flow fractionation [5], microstep [6] and pinched flow fractionation [7]. This work aims specifically to develop a simple, reusable, continuous, binary and deterministic separation method using microfluidics for erythrocytes and leukocytes separation without the need for lysing chemicals.

Human blood cells can be roughly classified into three groups: erythrocytes (RBC), leukocytes (WBC) and platelets. Leukocytes can be further classified into granulocytes, monocytes and lymphocytes. Table 1 compares the geometries and counts of erythrocytes and leukocytes in adults. Because of its shape, the height of a normal erythrocyte varies from 1.5 to $3.5 \mu \mathrm{m}$ [8], clearly smaller than the average leukocyte whose diameter falls between 7 and $20 \mu \mathrm{m}$. This difference in size enables us to use deterministic lateral displacement to separate the two types.

TABLE I: COMPARISON OF ERYTHROCYTES AND LEUKOCYTES $[8,10]$

\begin{tabular}{|l|cc|}
\hline & Erythrocyte & Leukocyte \\
\hline Cell shape & Biconcave disc & Sphere \\
Diameter average $(\mu \mathbf{m})$ & 6 & 10 \\
Diameter range $(\mu \mathbf{m})$ & $5-8$ & $7-20$ \\
Count $\left(\mathbf{1 0}^{\mathbf{9}} / \mathbf{L}\right)$ & $4,200-5,800$ & $4.5-11.0$ \\
\hline
\end{tabular}

The first such device was actually designed to separate submicron-sized beads or bacterial artificial chromosomes [9]. Here, we demonstrate the further application of this principle to the separation of much larger particles, including blood cells.

\section{DEVICE PRINCIPLE}

The device design involves a separation chamber filled by an array of cylindrical pillars. Each row of pillars has the same center-to-center distance $\lambda$ and edge-to-edge distance $\mathrm{d}$, and is staggered by a finite displacement from the previous row $\Delta \lambda$ (Fig. 1B). Because of small channel dimension (on the order of 10-100 $\mu \mathrm{m}$ ) and flow rate (on the order of $0.01-1 \mu \mathrm{l} / \mathrm{min}$ ), the Reynolds number is below 1 . Thus the flow inside the device can be treated as 2D laminar flow. For fully developed steady flow, each pillar having two stagnation points. The stagnation points of adjacent rows are rotated with respect to the net fluid flow direction because of the row shift. Separation lanes are defined by division lines, which are streamlines ending at stagnation points (Fig. 1C). We also define the critical separation size as twice the minimum distance between the edge of pillar and the nearest division line. For a given geometrical design, there is one corresponding critical separation size.

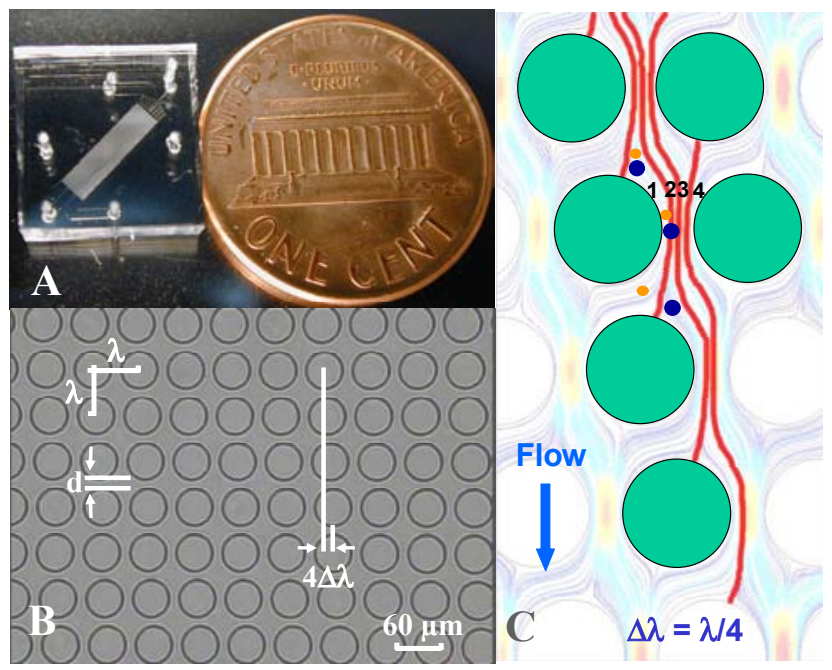

Fig. 1. (A) Device for blood separation. (B) Detailed device structure with geometrics labeled. (C) A small particle moves in zigzag mode and a large particle moves in displacement mode with four separation lanes for $\Delta \lambda=\lambda / 4$.

When the particles are not interacting with walls, we assume they will not change the flow pattern and will follow streamlines. The interactions between particles are also 
neglected for simplicity of the model. This is a good approximation for blood diluted over ten times, but might not be appropriate for undiluted blood. If the diameter of a particle is smaller than the critical separation size, it can follow a separation lane exactly resulting in a zigzag flow pattern which follows the net fluid flow direction over a long distance. On the other hand, if the diameter of a particle is larger than the critical separation size, it flows in displacement mode, meaning it does not remain in one separation lane all the time, and changes between lanes as it is unable to make sudden turns around pillars. These particles flow diagonally and do not follow the net fluid flow direction.

\section{DEVICE DESIGN AND FABRICATION}

We chose PDMS (Sylgard 184, Dow Corning, MI) for the device because of its blood compatibility and ease of use. Devices are fabricated with DRIE-silicon molds and mounted on a glass slide by overnight baking at $80{ }^{\circ} \mathrm{C}$ (Fig. 1A). The channel height is $20 \mu \mathrm{m}$. The effective separation area measures $7 \mathrm{~mm}$ by $1.8 \mathrm{~mm}$ and consists of upstream and downstream regions. In each region, every consecutive row of obstacles is shifted horizontally by a fixed amount: $4 \mu \mathrm{m}$ for upstream, $6 \mu \mathrm{m}$ for downstream. In both regions, along each row, the center-to-center distance of obstacles $\lambda$ is 60 $\mu \mathrm{m}$ while the distance between them $\mathrm{d}$ is $14 \mu \mathrm{m}$. At inlet, the blood sample is introduced and focused by two sheath flows.

\section{DeViCE Simulation}

The flow pattern inside the device is simulated by solving 2D incompressible Navier-Stokes equation with FEMLAB. No-slip boundary conditions are used for the device walls. Also assumed are parallel velocity field profile at the inlet and zero pressure at the outlet. Fig 2 shows the simulated streamlines for the modeled device. The colors are coded for magnitude of the velocity field. The critical separation size determined by simulation is 7.1 $\mu \mathrm{m}$.

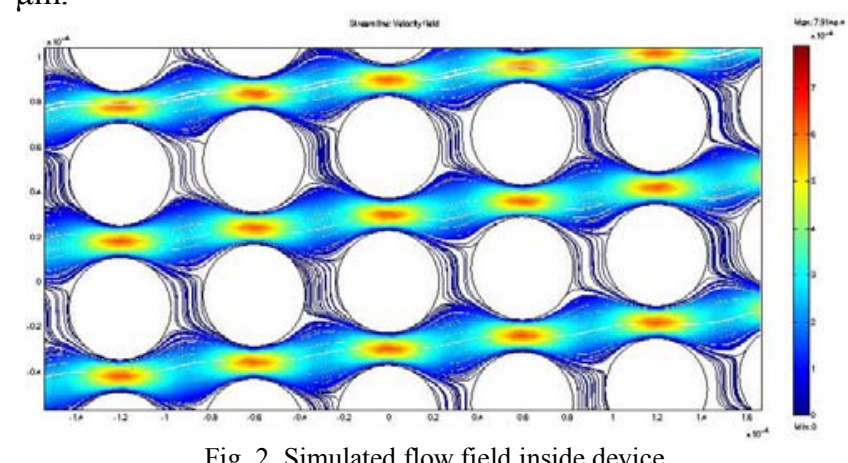

Fig. 2. Simulated flow field inside device.

\section{DeVice Calibration}

Experimentally, the device was first calibrated with polystyrene beads. Fluids were pumped into devices with syringe pumps (Pico Plus, Harvard Apparatus, MA). Central flow rate was kept at $0.2 \mu \mathrm{l} / \mathrm{min}$ and sheath flows were $1.2 \mu \mathrm{l} / \mathrm{min}$. Bead concentrations were on the order of $10^{3} / \mu 1$.

All beads $5 \mu \mathrm{m}$ (green) or $7 \mu \mathrm{m}$ (red) in diameter followed the "zigzag mode". In contrast, $10 \mu \mathrm{m}$ (red) diameter beads followed the "displacement mode". Finally, the $8 \mu \mathrm{m}$ diameter (clear) beads did not strictly follow either mode and exited between the $10 \mu \mathrm{m}$ and $7 \mu \mathrm{m}$ beads (Fig. 3 \& 4).

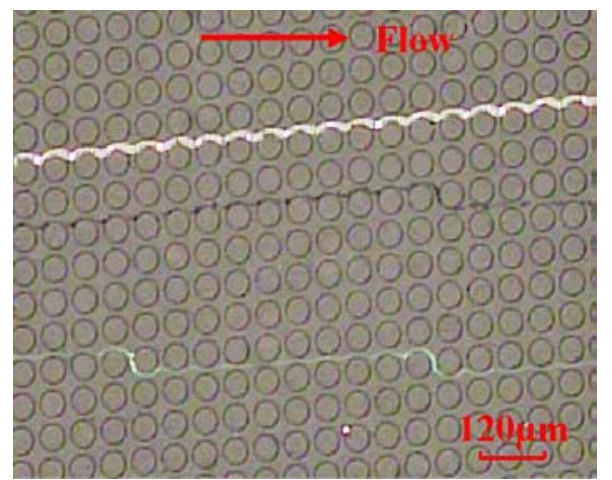

Fig. 3. Separation of $5 \mu \mathrm{m}$ (green), $8 \mu \mathrm{m}$ (clear) and $10 \mu \mathrm{m}$ (red) beads.

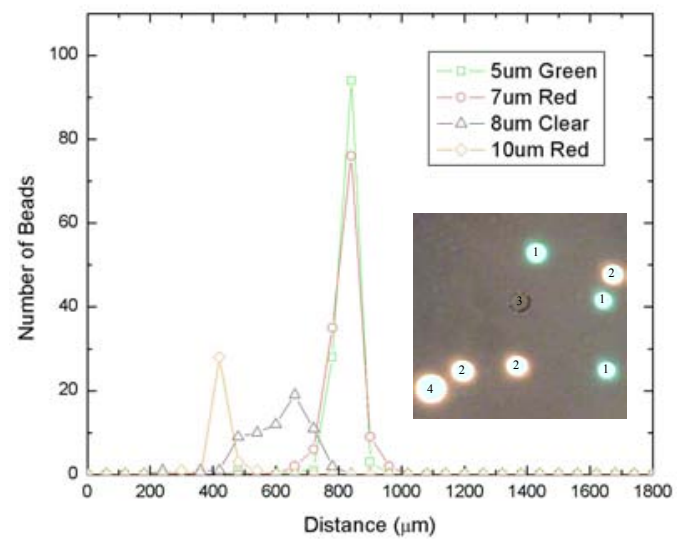

Fig. 4. Statistical analysis of beads separation. Inset is taken with fluorescent microscope. Beads with label 1,2,3 and 4 are $5 \mu \mathrm{m}, 7 \mu \mathrm{m}, 8 \mu \mathrm{m}$ and $10 \mu \mathrm{m}$ in diameter respectively.

To accurately measure the critical separation size and obtain a separation function, non-uniform fluorescent labeled polystyrene beads (Duke Scientific, CA, USA) are used under different flow rates (Fig. 5). Flow rate was found to have little effect on separation profile. The measured size distribution of the beads is given in Fig. 6. The separation function is calculated by a table lookup operation Fig. 7. The $\mathrm{S}$ shape of the separation function proves the binary nature of the separation, validating the use of the device for sorting applications. The experimentallydetermined critical separation size is approximately $8 \mu \mathrm{m}$, which agrees well with the simulation result.

\section{DeVICE TESTING}

Human blood cell separation was then demonstrated using a blood fraction of concentrated leukocytes diluted with Ficoll-Paque Plus (Amersham Biosciences, Sweden). Leukocytes were concentrated by repetitive centrifugation at 
$1500 \mathrm{rcf}$ (relative centrifugation force, equivalent to $\mathrm{g}$ ) for 10 minutes at $4^{\circ} \mathrm{C}$, then taking out top layer of supernatant and bottom layer of pellet. Ficoll-Paque Plus is a mixture of diatrizoate sodium and Ficoll 400. Ficoll 400 is a neutral, highly branched, hydrophilic polymer of sucrose.

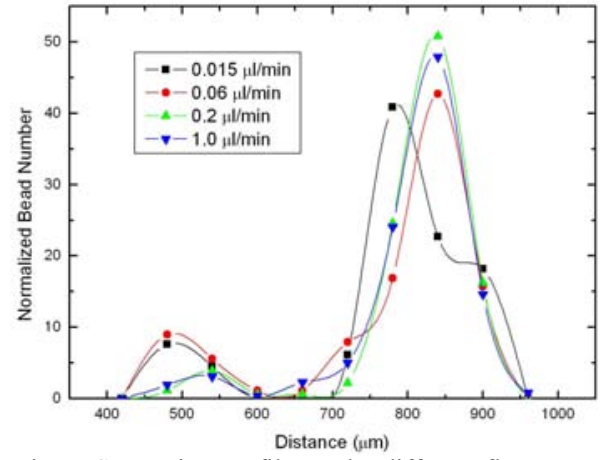

Fig. 5. Separation profiles under different flow rates.

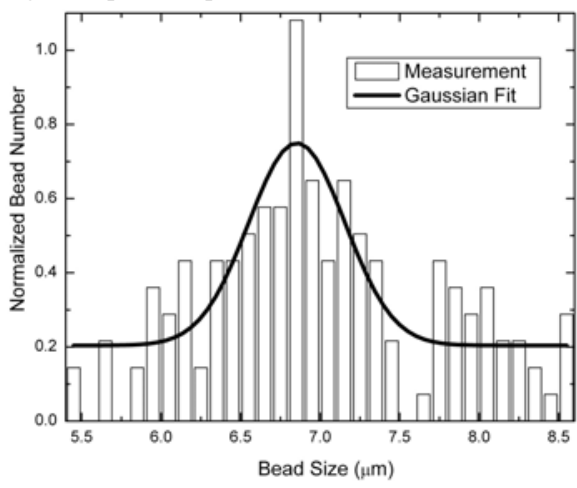

Fig. 6. Measure bead size distribution.

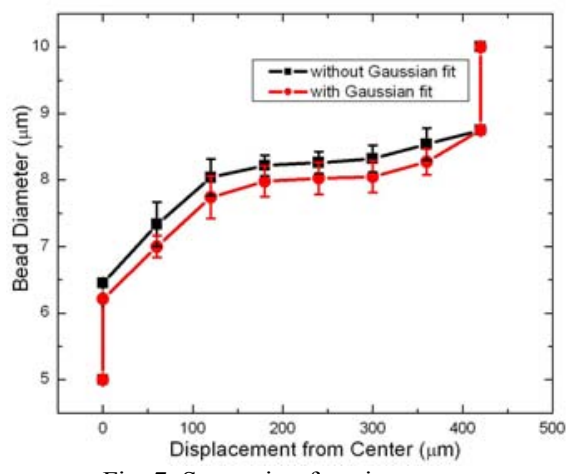

Fig. 7. Separation function curve.

Traditionally it has been used as a reagent to purify lymphocytes from human peripheral blood based on density gradient centrifugation. Its density is $1.077 \pm 0.001 \mathrm{~g} / \mathrm{ml}$, which is more close to blood cell density than aqueous saline. Diluting blood with Ficoll-Paque Plus enables us to run experiment over one hour without severe sedimentation.

The use of a hemacytometer yielded a measured leukocyte to erythrocyte ratio of 1:43. As shown in Fig. 8, two groups of cells following different flow patterns were identified. Statistical analysis concluded that the ratio of cells traveling displacement to those traveling zigzag was 1:38 (Fig. 9), very consistent with the leukocyte-toerythrocyte ratio.
We then performed tests using a sample of whole blood, diluted with Ficoll-Paque Plus. Because of the high erythrocyte-to-leukocyte ratio in natural blood, and limitations on flow rate and blood cell concentration, tests were run for over two hours in order to observe several leukocytes. Statistical analysis showed the ratio of cells traveling displacement to zigzag to be 1:502 (Fig. 10), which is in agreement with the known leukocyte-erythrocyte ratio. However, towards the end of such tests, several erythrocytes flowing in zigzag mode appeared in the lanes normally occupied by cells flowing in displacement mode. We believe such phenomena are caused by the increased amount of blood cells adhering to the obstacles after a long testing time.

To confirm that most leukocytes flow in displacement mode and estimate separation efficiency, we obtained leukocyte rich plasma by gravity sedimentation of erythrocytes in Wintrobe tubes and stained the leukocytes with acridine orange (Molecular Probes, OR, USA), before resuspension in Ficoll-Paque Plus. Acridine orange is a nuclear fluorescent dye. Erythrocytes, which have no nucleus, do not fluoresce, only leukocytes are stained and fluoresce. Fig. 11 shows the separation profile. As expected, most of the leukocytes are found to flow in displacement mode (Fig. 11 inset). The fraction of leukocytes with displacements overlapping erythrocytes is $9 \%$, indicating a small separation error or high leukocytes separation efficiency of $91 \%$.

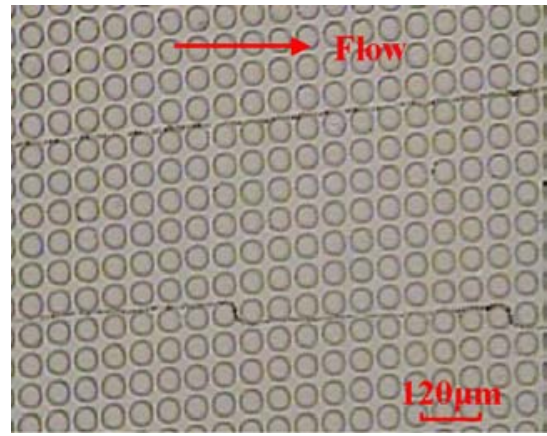

Fig. 8. Separation of leukocyte (top) from erythrocyte (bottom). Picture was generated by overlaying of multiple video frames.

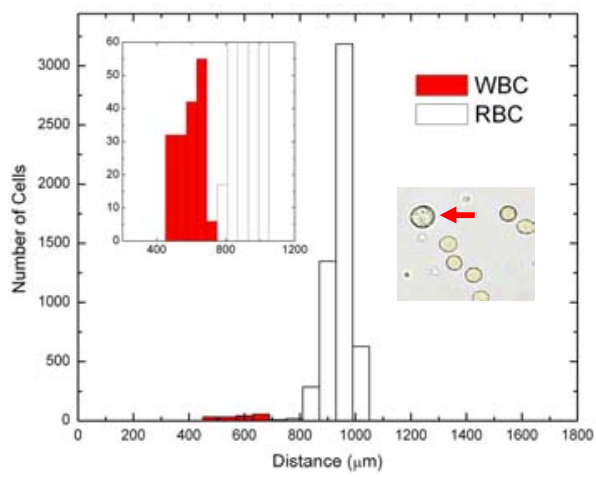

Fig. 9. Statistical analysis of separation of blood fraction of concentrated leukocytes. Left inset is a close up of leukocyte data. Right inset shows one leukocyte (arrow pointed) among erythrocytes. 


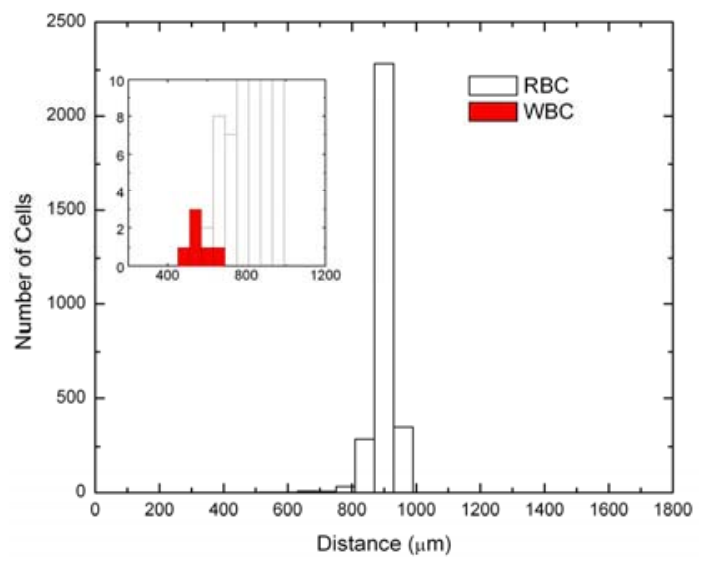

Fig. 10. Statistical analysis of separation of diluted whole blood sample.

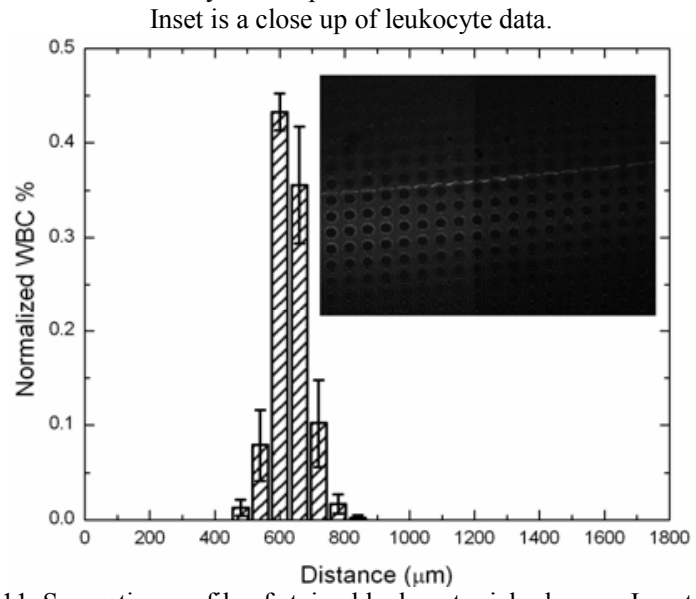

Fig. 11. Separation profile of stained leukocyte rich plasma. Insert is an event of leukocyte flow through the detection region.

\section{CONCLUSIONS AND DISCUSSION}

A MEMS device was demonstrated to separate leukocytes from erythrocytes based on deterministic lateral displacement principle. Fluorescent beads of $5 \mu \mathrm{m}, 7 \mu \mathrm{m}, 8$ $\mu \mathrm{m}$ and $10 \mu \mathrm{m}$ were successfully separated. Simulation of 2D laminar flow inside the device calculates velocity field and predicts the critical separation size for the current design to be $7.1 \mu \mathrm{m}$. The critical particle size for separation was shown to be around $8 \mu \mathrm{m}$ experimentally, which could be used for rough separation of blood cells. Ratio of blood cells following displacement mode to those in zigzag mode matches with leukocytes to erythrocytes ratio. Labeled leukocyte experiments confirm the separation of the two groups of cells is actually mainly erythrocytes and leukocytes. The efficiency for leukocyte separation is about $91 \%$.

In experiments, we observed polystyrene beads, which are more hydrophobic, were more likely to adhere to device surfaces then blood cells. Even in the downstream, polystyrene beads adhere readily to obstacles. Given the hydrophilic nature of cell surfaces, the hydrophobic property of PDMS makes it better to prevent severe cell adhesion.
It is also believed that in our devices, flow rate has a minor effect on separation because even at the highest flow rate $(2 \mu \mathrm{l} / \mathrm{min})$, the Reynolds number of the flow inside the device is at the order of $10^{-2}$ and the flows in our experiments are laminar. Therefore, it implies higher flow rate can be used to achieve fast separation.

Ongoing works focus on improving device performance and incorporate sensors downstream for blood counting. Effective separation area will be increased so as separation distance. Regions of different geometry design can be aligned in series so that separation of leukocytes into subtypes can be achieved. Coatings for device inner walls should be studied to prevent cell adhesion. Also it's interesting to study undiluted blood separation inside device and find the lowest working dilution level.

\section{ACKNOWLEDGEMENT}

This work is supported by NASA through National Space Biomedical Research Institute (NSBRI). The cooperative agreement number is NCC 9-58-317.

\section{REFERENCES}

[1] J. C. Giddings, "Field-flow Fractionation: Analysis of Macromolecular, Colloidal, and Particulate Materials," Science, vol. 260, pp. 1456-1465, 1993.

[2] J. Yang, Y. Huang, X. B. Wang, F. F. Becker, and R. C. Gascoyne, "Cell Separation on Microfabricated Electrodes Using Dielectrophoretic/gravitational Field Flow Fractionation," Analytical Chemistry, vol. 71, pp. 911-918, 1999.

[3] J. Cheng, E. L. Sheldon, L. Wu, A. Uribe, L. O. Gerrue, J. Carrino, M. J. Heller, and J. P. O'Connell, "Preparation and hybridization analysis of DNA/RNA from E-coli on microfabricated bioelectronic chips," Nature biotechnology, vol. 16, pp. 541-546, 1998.

[4] M. Berger, J. Castelino, R. Huang, M. Shah, and R. H. Austin, "Design of a Microfabricated Magnetic Cell Separator," Electrophoresis, vol. 22, pp. 3883 - 3892, 2001.

[5] Y. Jiang, M. N. Myers, and J. C. Giddings, "Separation behavior of blood cells in sedimentation field-flow fractionation," Journal Of Liquid Chromatography \& Related Technologies, vol. 22, pp. 1213-1234, 1999.

[6] S. Vankrunkelsven, D. Clicq, K. Pappaert, G. V. Baron, and G. Desmet, "A novel microstep device for the size separation of cells," presented at microTAS 2004, 2004.

[7] M. Nakashima, M. Yamada, and M. Seki, "Pinched flow fractionation (PFF) for continuous particle separation in a microfluidic device," presented at MEMS04, 2004.

[8] H. Kasdan, "personal communication," 2004.

[9] L. R. Huang, E. C. Cox, R. H. Austin, and J. C. Sturm, "Continuous Particle Separation Through Deterministic Lateral Displacement," Science, vol. 304, pp. 987-990, 2004.

[10] S. B. McKenzie, Clinical Laboratory Hematology: Prentice Hall, 2004. 